\title{
Transition Hausmannite Nanoparticles Embedded on Uniform Carbon Micro Spheres Synthesis for Electrochemical Examination
}

\author{
P. Devendran, S. Ezhil Arasi, R. Ranjithkumar, C. Sambathkumar, V. Manirathinam, \\ N.Nallamuthu, M. Krishna Kumar, A. Arivarasan, S. Asath Bahadur
}

\begin{abstract}
Carbon spheres wrapped by maghemite nanoparticles were synthesized through facile hydrothermal method. The structural parameters were analyzed through powder $x$-ray diffraction analysis. Functional groups were analyzed by Fourier transform infrared spectroscopic analysis. The prepared carbon spheres wrapped by maghemite nanoparticles morphology were investigated using scanning electron microscopic analysis. The elemental composition and distribution of elements were examined by energy dispersive spectroscopic technique with mapping. Redox property, charge discharge mechanism was done through cyclic voltammetry and galvanostatic charge-discharge studies.
\end{abstract}

Keywords-Maghemite nanoparticles, carbon spheres, hydrothermal, SEM, cyclic voltammetry, charge-discharge.

\section{INTRODUCTION}

In past few decades, nanotechnology has been great interest in vast area of applications for developing the nanosized materials. This can be achieved through various

Revised Manuscript Received on December 09, 2019.

* Correspondence Author

P. Devendran, Department of Physics, International Research Centre, Kalasalingam Academy of Research and Education, Krishnankoil626126. Tamil Nadu, India.

S. Ezhil Arasi, Department of Physics, International Research Centre, Kalasalingam Academy of Research and Education, Krishnankoil626126.Tamil Nadu, India.

R. Ranjithkumar, Department of Physics, International Research Centre, Kalasalingam Academy of Research and Education, Krishnankoil626126.Tamil Nadu, India.

C. Sambathkumar, Department of Physics, International Research Centre, Kalasalingam Academy of Research and Education, Krishnankoil626126. Tamil Nadu, India.

V. Manirathinam, Department of Physics, International Research Centre, Kalasalingam Academy of Research and Education, Krishnankoil626126.Tamil Nadu, India.

N.Nallamuthu, Department of Physics, International Research Centre, Kalasalingam Academy of Research and Education, Krishnankoil626126.Tamil Nadu, India.

M. Krishna Kumar, Department of Physics, International Research Centre, Kalasalingam Academy of Research and Education, Krishnankoil626126. Tamil Nadu, India.

A. Arivarasan, Department of Physics, International Research Centre, Kalasalingam Academy of Research and Education, Krishnankoil626126. Tamil Nadu, India.

S. Asath Bahadur, Department of Physics, International Research Centre, Kalasalingam Academy of Research and Education, Krishnankoil626126.Tamil Nadu, India. preparation techniques. Depending upon the size of nanomaterials their properties varies that are different from the macroscopic particles which lead to unique applications. This type of changes might be due to surface-volume ratio and quantum confinement effects [1-5].

The extraordinary physical and chemical properties of carbon spheres have attracted many researchers working in various areas. In recent days, the modifications in surface such as coating/wrapping inside and outside nanoparticles were of much interest. Many properties like optical, electronic, catalytic, and magnetic can be enhanced by wrapping the metal oxide nanoparticles onto the surface of carbon spheres. This makes the optics, electronics, catalysis and magnetism fields much wide with greater improvements [6-8].

Among various transition metal oxides, the iron oxide nanoparticles are magnetic inorganic material. It has many polymorphic structures in which the magnetite, maghemite and hematite are frequently formed. It has its own unique properties in various applications such as electrochemistry, electronics and magnetism, etc. Particularly, maghemite nanoparticles have wide variety of applications such as sensing, batteries, wastewater treatment, dye dehydration, catalysis, supercapacitors, etc [1, 9-11].

In this work, we have prepared carbon sphere wrapped by maghemite nanoparticles through hydrothermal route. Herein, iron (III) chloride and glucose has taken as starting material and have shown the electrochemically active nature of the prepared material.

\section{EXPERIMENTAL SECTION}

\section{A. Materials}

Iron (III) chloride hexa hydrate $\mathrm{Fe}(\mathrm{Cl})_{2} \cdot 6 \mathrm{H}_{2} \mathrm{O}$ was employed to prepare the maghemite nanoparticles and glucose for the carbon spheres and $\mathrm{NaOH}$ pellates. The chemicals with analytical grade were bought from Sisco Research Laboratories Pvt. Ltd., Mumbai, India. Maximum purity of about $99 \%$ was employed. De-ionized (DI) water was used as solvent for throughout reaction. KOH, PVDF, and NMP were purchased from Sigma Aldrich Pvt. Ltd for electrochemical studies.

\section{EXPERIMENTAL SECTION}




\section{A. Materials}

In order to prepare the sample, Glucose, $\mathrm{MnCl}_{2}, \mathrm{NaOH}$ and ethanol were purchased from Sisco Research Laboratories Pvt. Ltd., Mumbai, India. Analytical grade chemicals with high purity of $99 \%$ were used. Ultra-pure Millipore deionized (DI) water was used as a solvent for whole reaction. To do the electrochemical test, activated carbon (AC), potassium hydroxide $(\mathrm{KOH}), \quad \mathrm{N}$-methyl-2-pyrrolidinone $\left(\mathrm{C}_{5} \mathrm{H}_{9} \mathrm{NO}\right.$, NMP) and polyvinylidene fluoride $\left(\left(-\left(\mathrm{C}_{2} \mathrm{H}_{2} \mathrm{~F}_{2}\right)_{\mathrm{n}^{-}}\right), \mathrm{PVDF}\right)$ were used and it obtained from Sigma-Aldrich (Mumbai).

\section{B. Materials characterization}

$4 \mathrm{~g}$ of glucose were disolved in $30 \mathrm{ml}$ de-ionized water and stirred for $10 \mathrm{~min}$. The transperent solution was transferred into $50 \mathrm{ml}$ Teflon-lined autoclave and maintain temperature at $180{ }^{\circ} \mathrm{C}$ for $8 \mathrm{~h}$. Then allowed to room temp. the black pricipate was collected and dispersed under ultrasonic treatment for $10 \mathrm{~min}$. the product was recollect using centrifuged with 6000 RPM and washed several times with deionized water and ethanol. Finally, the prepared sample was dried at $80^{\circ} \mathrm{C}$ for $12 \mathrm{hrs}$ in a vacuum oven [12].

\section{Synthesis of carbon sphere wrapped by maghemite nanoparticles and modified electrode preparation}

The maghemite $\left(\mathrm{Fe}_{2} \mathrm{O}_{3}\right)$ nanoparticles wrapped on surface of carbon spheres were prepared by hydrothermal method. $0.1 \mathrm{M}$ of was dissolved in $120 \mathrm{ml}$ DI water. Separately, $0.1 \mathrm{M}$ of $\mathrm{Fe}(\mathrm{Cl})_{2} \cdot 6 \mathrm{H}_{2} \mathrm{O}, 0.5 \mathrm{~g}$ of carbon spheres and $0.1 \mathrm{M}(0.4 \mathrm{~g})$ of $\mathrm{NaOH}$ pellets were dispersed in DI water 50,50 and $15 \mathrm{ml}$ respectively. This three solutions were stirred up to $20 \mathrm{~min}$. to make a homogeneous dispersity. This two precursors $\left(\mathrm{Fe}(\mathrm{Cl})_{2} .6 \mathrm{H} 2 \mathrm{O}\right)$ and $\mathrm{NaOH}$ were drop by drop added in to dispersed carbon spheres solution and instantly, $\mathrm{Fe}_{2} \mathrm{O}_{3}$ precipitation were formed on the surface of carbon spheres. The reaction mixture was allowed to stir up to $3 \mathrm{~h}$ then transferred to teflon lined autoclave and maintained at 100 ${ }^{\circ} \mathrm{C}$ for $10 \mathrm{~h}$. The final product was washed several times with DI water and ethanol. The product was recollecting with centrifuged at $6000 \mathrm{RPM}$ and dried the final product at $80{ }^{\circ} \mathrm{C}$ for $12 \mathrm{~h}$ in a vacuum oven [12].

The modified electrode material was prepared by employing doctor blade technique. $\mathrm{Fe}_{2} \mathrm{O}_{3}$ NPs, conductive carbon with polyvinylidene fluoride as binder in the ratio of $85: 15: 5$, respectively. The mixture was grounded N-methylpyrrolidone as a solvent to make a slurry and it was coated on the surface of the Ni-foil $\left(1 \times 1 \mathrm{~cm}^{2}\right)$ and dried in an oven at 80 ${ }^{\circ} \mathrm{C}$ for $5 \mathrm{~h}$.

\section{Characterization techniques}

The synthesized carbon sphere wrapped by maghemite $\left(\mathrm{Fe}_{2} \mathrm{O}_{3}\right)$ nanoparticles were characterized using X-ray diffraction analysis, the structure of crystal formed were confirmed by X-ray diffraction analysis (XRD) by Bruker $\mathrm{X}$-ray diffractometer (D8 advance ECO) with monochromatic wavelength range $1.5406 \AA$ and $\mathrm{Cu}-\mathrm{K}_{\alpha}$ radiation source. Scanning Electron Microscope (SEM) with Energy Dispersive Spectroscopy (EDS) and mapping was used to observe the morphology, elemental distribution of carbon sphere wrapped nanoparticles using ZEISS-EVO 18 Research, Japan, and sample Fourier transform infrared spectrometer (FTIR) was employed using a Shimadzu (IR Tracer-100) spectrophotometer within the range of 4000-400 $\mathrm{cm}^{-1}$ using $\mathrm{KBr}$ pellet system. Electrochemical workstation with three electrode system ( $\mathrm{CH}$ instrument $6008 \mathrm{E})$ was used to investigate the redox nature and impedance analysis.

\section{RESULTS AND DISCUSSION}

\section{A. X-ray diffraction-structural analysis}

Fig.1. shows a XRD pattern of carbon sphere wrapped by maghemite $\left(\mathrm{Fe}_{2} \mathrm{O}_{3}\right)$ nanoparticles. The sharp edges and broadening of peaks implies that the prepared material were nano-crystallized with small size in nanoscale range. $30.12^{\circ}$, $35.67^{\circ}, 43.36^{\circ}, 53.84^{\circ}, 57.29^{\circ}, 62.64^{\circ}, 71.42^{\circ}$, and $74.52^{\circ}$ were the diffracted peaks matched well with JCPDS number 39-1346. These diffracted peaks were indexed to the hkl planes (220), (311), (400), (422), (511), (440), (620), and (533) respectively [13, $14 \& 15]$. It confirmed the prepared nanoparticles were cubic structure of $\mathrm{Fe}_{2} \mathrm{O}_{3}$ nanocrystals. The broadened line was influenced by crystallite size of the prepared nanoparticles. The crystallite size was determined from Scherrer formula given in equation (1) and hence calculated the average grain size were found to be $\sim 22 \mathrm{~nm}$.



Fig. 1. XRD pattern of prepared carbon sphere wrapped by maghemite nanoparticels

\section{B. FTIR spectral studies.}

The FTIR spectrum of prepared material was shown in Fig. 2. The spectral range was fixed between $4000-400 \mathrm{~cm}^{-1}$. 


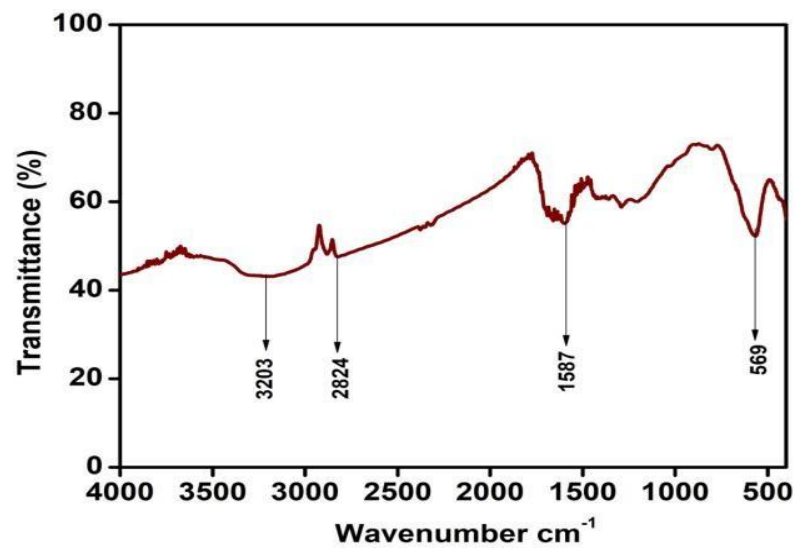

Fig. 2.FTIR spectrum of carbon sphere wrapped by maghemite nanoparticles

The stretching vibration of Fe-O was found in $569 \mathrm{~cm}^{-1}$. The strong peak near $1587 \mathrm{~cm}^{-1}$ indicated presence of $\mathrm{C}=\mathrm{O}$. $\mathrm{C} \equiv \mathrm{C}$ bond vibrations were observed in the peak $2824 \mathrm{~cm}^{-1}$. The broad peak at $3203 \mathrm{~cm}^{-1}$ shows the $\mathrm{O}-\mathrm{H}$ bond formation which might be due to the absorption from the surrounding as it contains mesoporous structure.

\section{SEM- Morphological studies.}

It was evident from the name that the prepared carbon spheres wrapped by maghemite nanoparticles were observed to be in sphere morphology. The smooth surface with the maghemite nanoparticles wrapping were evident from lower magnifications.



Fig. 3.SEM images of maghemite nanoparticles wrapped carbon sphere
In higher magnification of around $200 \mathrm{~nm}$ the maghemite nanoparticles wrapping on the surface of the carbon sphere were clearly observed [12].

\section{EDS with mapping analysis}

A spectrum recorded explains the purity of the prepared carbon sphere wrapped by maghemite nanoparticles. EDS spectrum with mapping images were shown in Fig. 4. The weight percentage was shown as inset in the Fig. 4. The mapping images clearly indicated that prepared carbon sphere wrapped by maghemite nanoparticles were even in distribution.



Fig. 4.EDS spectrum and mapping of prepared carbon sphere wrapped by maghemite nanoparticles

\section{E. Electrochemical and Cyclic voltammetry analysis}

The redox property of the prepared carbon sphere wrapped by maghemite nanoparticles were investigated by cyclic voltammetry studies. Fig. 5. reveals the CV graph of prepared nanoparticles with different scan rate. The potential window was fixed between 0.2 to $0.6 \mathrm{~V}$. A pair of redox peaks for various scan rates implies the pseudocapacitance nature. As the characteristic peaks were observed in both cathodic and anodic region, the prepared material has been confirmed to have the capacitance nature $[1,12 \& 15]$.

\section{F. Chronopotentiometric analysis}

The galvnostatic charge-discharge curve of the prepared maghemite nanoparticles wrapped carbon sphere were shown in Fig. 6. The potential window was fixed to 0 to $0.5 \mathrm{~V}$. The curve was recorded with the current $0.5 \mathrm{~mA} / \mathrm{Cm}^{2}$. It states that the specific capacitance and storage capacity of material [10-12, 16]. 


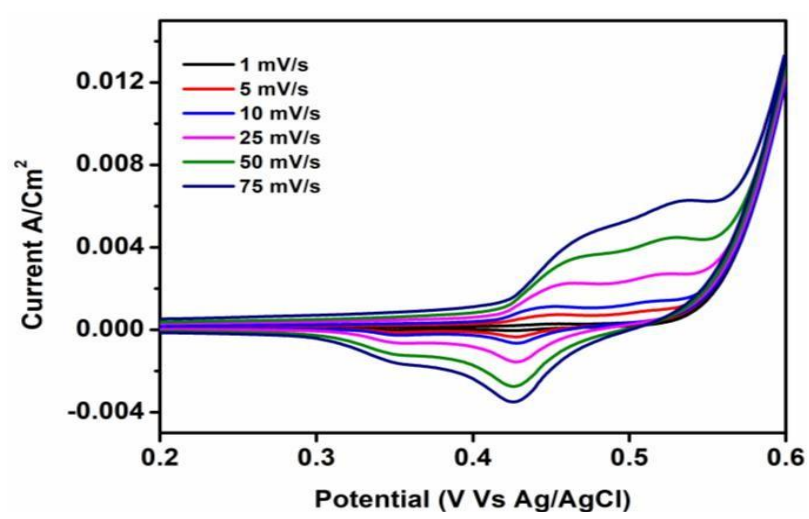

Fig. 5.CV graph of carbon sphere wrapped by maghemite nanopaticles

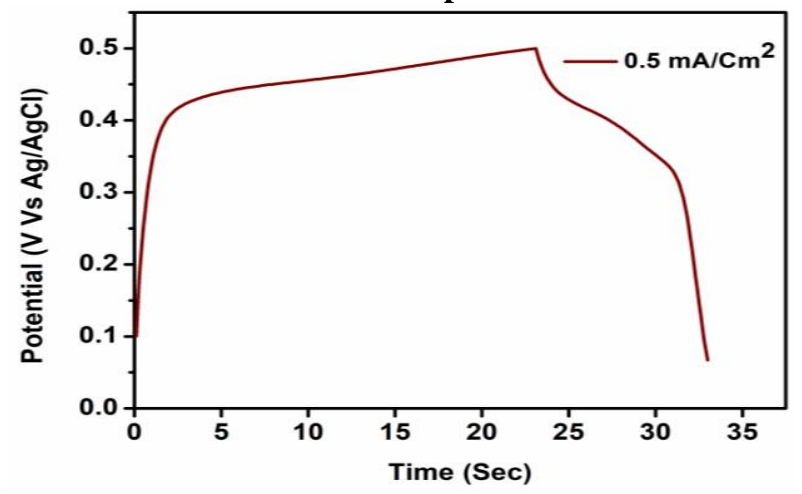

Fig. 6.GCD curve of carbon sphere wrapped by maghemite nanopaticles.

\section{CONCLUSION}

In summary, The carbon sphere wrapped maghemite nanoparticles were successfully prepared by effective hydrothermal method. The XRD pattern shows that the prepared mag material was cubic structure with grain size of nm. The carbon-carbon bond vibrations and metal oxide vibrations were confirmed in FTIR spectrum. These results have been co-ordinated with the EDS spectrum as the atomic and elemental weight percentage was almost same with the elemental compositions. The morphology of the prepared nanoparticles was found to have spheres shape with the maghemite nanoparticles wrapped on the surface. This was in corresponded with the mapping images as the distribution of nanoparticles were even. The redox behaviour was investigated and found to have two pair of redox peaks in anodic and cathodic region. The charge-discharge process states that the prepared material could be a pesudocapacitive material with good energy storage property.

\section{ACKNOWLEDGMENT}

The author P.D and all co-authors are very grateful to IRC, Kalasalingam Academy Research and Education (KARE) for providing and facilities.

\section{REFERENCES}

1. P. Devendran, T. Alagesan, A. Manikandan, S. Asath Bahadur, M. Krishna Kumar, S. Rathinavel, K. Pandian, "Sonochemical Synthesis of Bi2S3 Nanowires using Single Source Precursor and Study of Its Electrochemical Activity" Nanosci. and Nanotechnol. Lett. 2016, 8, 1-6.
2. P. Devendran, T. Alagesan, T. R. Ravindran, K. Pandian, Synthesis of spherical CdS quantum dots using cadmium diethyldithiocarbamate as single source precursor in olive oil medium, Current Nanoscience, 2014, 10, 302-307.

3. P. Devendran, T. Alagesan, K. Pandian, "Single pot microwave synthesis of CdS nanoparticles in ionic liquid and their photocatalytic application" Asian Journal of Chemistry, Sup. Issue, 2013, 25, S79S82.

4. A. Shameem, P. Devendran, V. Siva, M. Raja, A. Manikandan, S. Asath Bahadur, Preparation and Characterization of Nanostructured $\mathrm{CdO}$ thin films by SILAR method for Photocatalytic Application, J. of inorganic and organometallic poly. and mat., 2017, 27, 692-699.

5. R. Packiaraj, P. Devendran, S. Asath Bahadur, N. Nallamuthu, Structural and electrochemical studies of Scheelite type BiVO4 nanoparticles: synthesis by simple hydrothermal method, J. of Materials Science: Materials in Electronics (2018) 29:13265-13276.

6. Yair, K.; Marcus, R.; Emanuel, K.; Lars, B.; Alexander, K.; Stefan, K.; Gleb, Y., High-Rate Electrochemical Capacitors Based on Ordered Mesoporous Silicon Carbide-Derived Carbon.ACS Nano 2010, 4, 1337-1344.

7. Pan, S.; Lin, H.; Deng, J.; Chen, P.; Chen, X.; Yang, Z.; Peng, H., Novel Wearable Energy Devices Based on Aligned Carbon Nanotube Fiber Textiles. Adv. Energy Mater. 2015, 5, 1401438.

8. Zhang, Y.; Zhao, C.; Ong, W. K.; Lu, X., Ultrafast-Freezing-Assisted Mild Preparation of Biomass-Derived, Hierarchically Porous, Activated Carbon Aerogels for High-Performance Supercapacitors. ACS Sustain. Chem. Eng. 2018, 7, 403-411.

9. K. Seevakan, A. Manikandan, P. Devendran, A. Shameem, T. Alagesan, Microwave combustion synthesis, magneto-optical and electrochemical properties of NiMoO4 nanoparticles for super capacitor application, Ceramics International 44 (2018) 1387913887.

10. K. Seevakan, A. Manikandan, P. Devendran, A. Baykal, Y. Slimani, T. Alagesan Structural, morphological and magneto-optical properties of CuMoO4 electrochemical nanocatalyst as supercapacitor electrode, Ceramics International 44 (2018) 20075-20083.

11. K. Seevakan, A. Manikandan, P. Devendran, S. Arul Antony, T. Alagesan, One-pot synthesis and characterization studies of iron molybdenum mixed metal oxide ( $\mathrm{Fe} 2(\mathrm{MoO} 4) 3)$ nano - nanocatalysts, Advanced Science, Engineering and Medicine, 2016, 8, 1-7.

12. P. Devendran, T. Alagesan, K. Pandian, Synthesis and characterization of Bi2S3 nanorods decorated on carbon sphere and study its electrochemical application, Advanced Materials Research, 2014, 938, 215-220.

13. A. M. Prodan, S. L. Iconaru, C. M. Chifiriuc, C. Bleotu, C. S. Ciobanu, M. Motelica-Heino, S. Sizaret, D. Predoi, Magnetic Properties and Biological Activity Evaluation of Iron Oxide Nanoparticles, Journal of Nanomaterials 2013 (893970) 1-7.

14. M. Aliahmad, N. Nasiri Moghaddam, Synthesis of maghemite $(\gamma-$ $\mathrm{Fe} 2 \mathrm{O} 3$ ) nanoparticles by thermal-decomposition of magnetite (Fe3O4) nanoparticles, Materials Science-Poland, 2013, 31( 2) 264-268.

15. B. P. Singh, Arun kumar, H. I. A-Martinez, C. A. V. Olivencia, S. M. Tomar, Synthesis, Charactrization and electrocatalytic ablity of $\mathrm{Fe} 2 \mathrm{O} 3$ anoparticles for sensing acetaminophen, Indian Journal pf pure \& Appliced physics, 2017, 55, 722-728.

16. R. Ranjithkumar, S. Ezhil Arasi, S. Sudhahar, N. Nallamuthu, P. Devendran, P. Lakshmanan, M. Krishna Kumar, Enhanced electrochemical studies of $\mathrm{ZnO}-\mathrm{CNT}$ nanocomposite for supercapacitor devices, Physica B Vol.568 (2019) pp.51-59. 